\title{
Informative Inter Simple Sequence Repeat (ISSR) primers for genetic analysis of coconut (Cocos nucifera $\mathrm{L}$.) germplasm
}

\author{
R. Manimekalai ${ }^{1}$
}

\begin{abstract}
Inter Simple Sequence Repeat (ISSR) markers are versatile and used in a number of applications viz. genetic diversity estimation, phylogenetic relationship and gene tagging in different crops. In coconut, the ISSR markers are being reported here for the first time. In the present paper, thirty-five primers targeting to amplify the inter microsatellite regions were screened using thirty different coconut germplasm accessions. The ISSR primers were evaluated based on polymorphism information content and marker index. Out of 35 primers screened, 19 primers produced clear amplification pattern. The polymorphism information content varied between 0.019 and 0.386 , whereas, the marker index ranged from 0.019 to 5.673 among the primers. Based on the high marker index, five and ten primers were selected. The similarity matrices were constructed separately for five, ten and 19 primers using NYSYS software and the correlation was tested using Mantel's test. There was high correlation between five and ten primers with 19 primers. Hence, the primers with higher marker index ( 5 and 10 primers) were regarded as informative primers. These informative primers can be used to develop more polymorphic markers in coconut for rapid screening of germplasm materials.
\end{abstract}

Key words: ISSR, coconut germplasm, molecular markers.

${ }^{1}$ Scientist (Biotechnology), Crop Improvment Division, Central Plantation Crops Research Institute, Kasaragod-671 124, Kerala, India. 


\section{Introduction}

Inter-Simple Sequence Repeats (ISSR) technique is a PCR based method, which involves amplification of DNA segment present at an amplifiable distance in between two identical microsatellite repeat regions oriented in opposite direction (Zietkiewicz et al., 1994). The technique uses microsatellites, usually 16-25 bp long as primers in a single primer PCR reaction targeting multiple genomic loci to amplify mainly the inter-SSR sequences of different sizes. The microsatellite repeats used as primer can be di-nucleotide, tri-nucleotide, tetranucleotide or penta-nucleotide.

ISSR markers are highly polymorphic and are used in studies on genetic diversity, phylogeny, gene tagging, genome mapping and evolutionary biology (Reddy et al., 2002). ISSR PCR is a technique which overcomes the problems like low reproducibility of RAPD, high cost of AFLP, the need to know the flanking sequences to develop species specific primers for SSR polymorphism (Goodwin et al., 1997). ISSR segregate mostly as dominant markers following simple Mendelian inheritance (Wang et al., 1998). However, they have also been shown to segregate as codominant markers in some cases thus enabling distinction between homozygote and heterozygote (Sankar and Moore, 2001). ISSR amplification is a relatively recent technique which can differentiate closely related genotypes.

The technique combines most of the benefits of AFLP and microsatellite analysis with the universality of RAPD. ISSR have high reproducibility possibly due to the use of longer primers (16 - 25 mers) as compared to RAPD primers (10 mers) which permits the subsequent use of annealing temperature $\left(45-60{ }^{\circ} \mathrm{C}\right)$ leading to higher stringency. The limitation of this marker is that the fragments with the same mobility originate from non-homologous regions, which can contribute to some distortion in the estimates of genetic similarities. No reports are available for coconut about the usefulness of ISSR markers. Effectiveness of ISSR primers were evaluated interms of
Polymorphism Information Content (PIC) and Marker Index (MI).

\section{Polymorphism Information Content}

Polymorphism information content (PIC) is a quantification of the number of alleles or bands that a marker has and the frequency of each of the alleles or bands in the population of OTUs in the study. A marker with fewer bands has less power to distinguish several OTUs; also, alleles present in a low frequency have less power to distinguish the OTUs. Hence, a higher PIC is assigned to a marker with many alleles and with alleles present at roughly equal proportions in the population (Powell et al., 1996; Smith et al., 1997). For multi-locus markers like ISSR, marker index reveals usefulness of the primers in detecting higher polymorphism.

\section{Materials and methods}

Thirty coconut germplasm accessions were used to screen the primers. DNA was extracted by following the method of Upadhyay et al. (1999).

\section{ISSR analysis}

Thirty-five ISSR primers obtained from University of British Columbia (UBC 807, UBC 809, UBC 810, UBC 812, UBC 814, UBC 815, UBC 816, UBC 818, UBC 819, UBC 822, UBC 823, UBC 824, UBC 825, UBC 826, UBC 827, UBC 830, UBC 834, UBC 835, UBC 836, UBC 841, UBC 844, UBC 845, UBC 854, UBC 855, UBC 856, UBC 857, UBC 858, UBC 861, UBC 863, UBC 864, UBC 868, UBC 869, UBC 889, UBC 895 , UBC 898) were used in the study.

Amplification reactions were carried out in $10 \mu \mathrm{l}$ volume containing $30 \mathrm{ng}$ of template DNA, $200 \mu \mathrm{M}$ of each dNTPs, $0.36 \mathrm{U}$ of Taq polymerase (Bangalore Genei Pvt. Ltd., India) and $20 \mu \mathrm{M}$ of ISSR primer (University of British Columbia, Canada). Amplifications were performed in a Thermo Cycler (MJ Research Inc., USA) programmed for an initial denaturation at $94^{\circ} \mathrm{C}$ for 5 min., 40 cycles of 1 min. denaturation at $94^{\circ} \mathrm{C}, 1 \mathrm{~min}$. annealing at 
$50^{\circ} \mathrm{C}$ and $2 \mathrm{~min}$. extension at $72^{\circ} \mathrm{C}$ and a final extension of $7 \mathrm{~min}$. at $72^{\circ} \mathrm{C}$. The PCR products were subjected to electrophoresis through a $2.00 \%$ agarose gel using $1 \mathrm{X}$ TBE buffer at 90 volts for $2 \mathrm{~h}$ in Bio-Rad submarine electrophoresis unit. The ethidium bromide stained gels were documented using the Alpha Imager TM 1200-Documentation and Analysis system (Alpha Innotech Corporation, USA).

\section{Data analysis}

Only the clear, unambiguous bands were considered for scoring. Each band was considered to be a single locus. Data were scored as " 1 " for the presence and " 0 " for the absence of a DNA band of each accession. DNA band size was estimated by comparing the DNA bands with a $1 \mathrm{~Kb}$ DNA ladder or lambda DNA Eco RI/Hind III double digest (MBI Fermentas, Lithuania). The average polymorphism information content (PIC) and marker index (MI) were calculated by applying the formulas given by Powell et al. (1996) and Smith et al. (1997):

$$
\mathrm{PIC}=1-\sum_{\mathrm{i}=1}^{\mathrm{n}} \mathrm{fi}^{2}
$$

where fi is the frequency of the $i^{\text {th }}$ allele. The number of alleles refers to the number of scored bands. The frequency of an allele was obtained by dividing the number of accessions where it was found by the total number of accessions. The PIC value provides an estimate of the discriminating power of a marker. Marker indices were calculated for each primer as the product of PIC and the number of polymorphic bands. The MI reveals the informativeness of the primers. If the MI is higher, the primer is more informative.

\section{Matrix comparison}

The similarity matrices produced by total primers and a set of primers (5 and 10) were compared by the MXCOMP routine of NTSYS pc. The normalized Mantel statistic Z (Mantel, 1967) was used to determine the level of association between the matrices.

\section{Results and discussion}

Out of 35 primers screened, 19 primers gave clear amplification profiles and produced a total of 199 markers across 30 coconut accessions tested. Out of 199 markers, 154 markers were polymorphic. The number of total markers for each primer varied between five (UBC 814) to 17 (UBC 854) with an average of 10.5 markers per primer. The number of polymorphic markers ranged from one (UBC 864 ) to 15 (UBC 855) with a mean of 8.10. The per cent polymorphism ranged from 16.6 (UBC 864 ) to 100 (UBC 855 and UBC 827) with a mean of 77.39. The amplification product size ranged from $206 \mathrm{bp}$ (UBC 834) to $2618 \mathrm{bp}$ (UBC 815).

The ISSR marker profiles generated by the primer UBC 855 is shown in Fig. 1.

\section{MI and PIC}

In the present investigation, marker index (MI) was calculated which reveals the amount of information that can be obtained from a particular primer. Higher the MI, more the informativeness of the primer. The marker index among ISSR primers was in the range of 0.0195.673. The primer UBC 855 recorded the highest marker index (5.673) followed by UBC 854 (4.563), UBC 815 (3.635), UBC 808 (3.147) and UBC 810 (3.085), and it was the lowest for the primer UBC $864(0.019)$ with a mean of 2.279. The PIC value was the highest for the ISSR primer UBC 810 (0.386) followed by UBC 855 (0.378), UBC 827 (0.343), UBC 856 (0.335) and UBC 854 (0.326). The mean PIC value among ISSR primers was 0.255 (Table 1).

ISSR primers were classified as I five and II five informative primers based on their higher marker Index (Table 2). Among the I five informative primers, UBC 855 recorded the highest number of polymorphic markers (15). I five informative primers produced a total of 61 polymorphic markers. UBC 855 recorded the highest value for marker index (5.673). Among the II five informative primers, UBC 889 recorded number of polymorphic markers (10). 


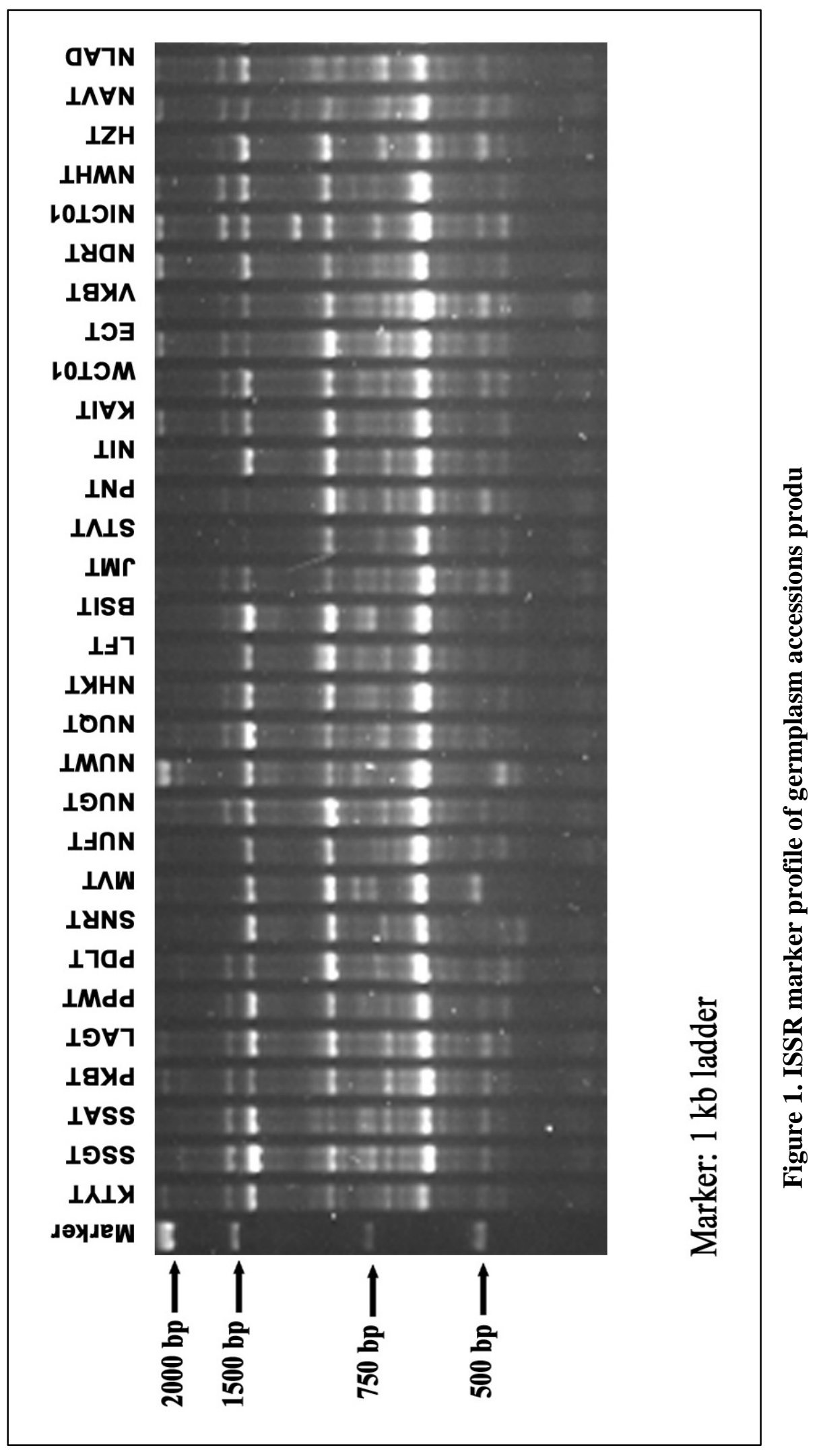


Table 1. Details of polymorphism information content (PIC) and marker index (MI) among ISSR primers

\begin{tabular}{|c|c|c|}
\hline Primer & $\begin{array}{c}\text { Polymorphism information } \\
\text { content (PIC) }\end{array}$ & Marker index (MI) \\
\hline UBC 807 & 0.221 & 1.766 \\
UBC 808 & 0.262 & 3.147 \\
UBC 810 & $\mathbf{0 . 3 8 6}$ & 3.085 \\
UBC 814 & 0.171 & 0.513 \\
UBC 815 & 0.303 & 3.635 \\
UBC 823 & 0.286 & 2.574 \\
UBC 824 & 0.274 & 2.193 \\
UBC 827 & 0.343 & 2.060 \\
UBC 834 & 0.198 & 1.778 \\
UBC 835 & 0.259 & 1.813 \\
UBC 836 & 0.319 & 2.550 \\
UBC 841 & 0.197 & 1.575 \\
UBC 842 & 0.174 & 0.872 \\
UBC 854 & 0.326 & 4.563 \\
UBC 855 & 0.378 & $\mathbf{5 . 6 7 3}$ \\
UBC 856 & 0.335 & 2.680 \\
UBC 864 & $\mathbf{0 . 0 1 9}$ & $\mathbf{0 . 0 1 9}$ \\
UBC 868 & 0.169 & 0.506 \\
\hline
\end{tabular}

Table 2. List of ISSR informative primers and number of polymorphic markers and its marker indices (MI)

\begin{tabular}{|c|c|c|c|}
\hline S.No. & Primer & Polymorphic markers (No.) & MI \\
\hline 1 & UBC 855 & $\mathbf{1 5}$ & $\mathbf{5 . 6 7 3}$ \\
2 & UBC 854 & 14 & 4.563 \\
3 & UBC 815 & 12 & 3.635 \\
4 & UBC 808 & 12 & 3.147 \\
5 & UBC 810 & 8 & 3.085 \\
\hline & Total & $\mathbf{6 1}$ & \\
\hline 6 & UBC 856 & 9 & $\mathbf{2 . 6 8 0}$ \\
7 & UBC 823 & 8 & 2.574 \\
8 & UBC 836 & 10 & 2.550 \\
9 & UBC 889 & 8 & 2.311 \\
10 & UBC 824 & $\mathbf{4 3}$ & 2.193 \\
\hline \multicolumn{2}{|r}{} \\
\hline
\end{tabular}


II five informative primers produced a total of 43 polymorphic markers. UBC 856 recorded the highest value for marker index (2.680). Overall, 10 informative ISSR primers (I five + II five) produced 104 polymorphic markers.

\section{Comparison of similarity matrices}

The similarity matrix based on five primers (I five informative primers) and ten primers (I + II informative primers) were compared with similarity matrix of 19 primers using Mantel's test in the software NTSYS pc. The results showed that high correlation existed between 19 primers with ten informative primers (' $r$ ' value $=0.941$ ) and five informative primers (' $r$ ' value $=0.901$ ). Absolute correlation was found between five and ten informative primers ('r' value $=1.000$ ).

As high correlation was found between the similarity matrices of informative primers to that of 19 primers, the genetic similarity estimate produced by the informative primers will be comparable with that of 19 primers. Hence, informative primers alone can be used for large scale screening of coconut germplasm.

The following five primers with higher marker index viz., UBC 855 (5.673), UBC 854 (4.563), UBC 815 (3.635), UBC 808 (3.147) and UBC 810 (3.085) have been selected as I five informative primers. These informative primers would be very useful in future for large scale screening of germplasm for higher polymorphism, to determine the genetic diversity in coconut, and prove useful in establishing core collection of coconut germplasm.

\section{Acknowledgement}

Author thanks Director, Dr. V. Rajagopal, CPCRI, Kasaragod for the necessary facilities to carry out the work.

\section{References}

Goodwin, I.D., Aitken, E.A.B. and Smith, L.W. 1997. Application of inter simple sequence repeat (ISSR) markers to plant genetics. Electrophoresis 18: 1524-1528.

Mantel, N. 1967. The detection of disease clustering and a generalized regression approach. Cancer Res. 27: 209-220.

Powell, W., Morgane, M., Andre, C., Hanafey, M., Vogel, J., Tingey, S.V. and Rafalski, A. 1996. The comparison of RFLP, RAPD, AFLP and SSR markers for germplasm analysis. Mol. Breed. 2: 225238.

Reddy, M.P., Sarla, N. and Siddiq, E.A. 2002. Inter simple sequence repeat (ISSR) polymorphism and its application in plant breeding. Euphytica 128: 9-17.

Sankar, A.A. and G.A. Moore. 2001. Evaluation of inter simple sequence repeat analysis for mapping in citrus and extension of genetic linkage map. Theor. Appl. Genet. 102: 206-214.

Smith J.S.C., Chin, E.C.L., Shu, H., Smith, O.S., Wall, S.J., Senior, M.L., Mitchell, S., Kresovich, S.E. and Ziegle, J. 1997. An evaluation of SSR loci as molecular markers in maize (Zea mays L.): Comparison with data from RFLPs and pedigree. Theor. Appl. Genet. 95: 163-173.

Upadhyay, A., Parthasarathy, V.A., Seema, G. and Karun, A. 1999. An efficient method of DNA extraction from coconut. Agrotropica 11: 35-38.

Wang, G., Mahalingan, R. and Knap, H.T. 1998. (C-A) and (G-A) Anchored Simple Sequence Repeats (ASSR) generated polymorphism in soybean Glycine max (L.) Theor. Appl. Genet. 96: 1086-1096.

Zietkiewicz, E., Rafalski, A. and Labuda, D. 1994. Genome fingerprinting by simple sequence repeat (SSR) anchored polymerase chain reaction amplification. Genome 20: 176-183. 